\title{
Variants of P Colonies with Very Simple Cell Structure
}

\author{
Lucie Ciencialová, Erzsébet Csuhaj-Varjú, Alica Kelemenová, György Vaszil
}

Lucie Ciencialová, Alica Kelemenová

Institute of Computer Science, Faculty of Philosophy and Science, Silesian University in Opava

Bezručovo nám. 13, 74601 Opava, Czech Republic

E-mail: \{lucie.ciencialova, alica.kelemenova\}@ fpf.slu.cz

Erzsébet Csuhaj-Varjú, György Vaszil

Computer and Automation Research Institute of the Hungarian Academy of Sciences

Kende utca 13-17, 1111 Budapest, Hungary

E-mail: \{csuhaj, vaszil\}@ sztaki.hu

Erzsébet Csuhaj-Varjú

Department of Algorithms and Their Applications, Faculty of Informatics, Eötvös Loránd University

Pázmány Péter sétány 1/c, 1117 Budapest, Hungary

Alica Kelemenová

Department of Computer Science, Catholic University Ružomberok

Nám. A. Hlinku 56, 03401 Ružomberok, Slovakia

Received: April 5, 2009

Accepted: May 30, 2009

\begin{abstract}
We study two very simple variants of P colonies: systems with only one object inside the cells, and systems with insertion-deletion programs, so called $\mathrm{P}$ colonies with senders and consumers. We show that both of these extremely simple types of systems are able to compute any recursively enumerable set of vectors of non-negative integers.
\end{abstract}

Keywords: P systems, colonies, P colonies, register machines

\section{Introduction}

P colonies form a class of abstract computing devices modeling a community of simple agents acting and evolving in a shared environment. They were introduced in [5] as very simple membrane systems, similar in simplicity and architecture to so called colonies of formal grammars. (See [7] for more information on membrane systems and $[2,4]$ for details on grammar systems theory.)

A P colony consists of a collection of cells, each having a number of objects inside and an associated set of rules through which it can process these objects. Communication between the cells is only possible indirectly through the environment which is common to all of them.

The capabilities of the computing agents are very restricted, and the number of objects present inside a cell during the functioning of the system is previously fixed: it is usually one, two or three. The rules are also of a very simple form. As we will see, they allow the transformation of objects inside the cells and the transportation of objects between the cells and the environment. The rules are grouped into programs. A program contains exactly as many rules, as the number of objects allowed to be present inside the cell. The rules of the programs are applied to the objects inside the associated cells in parallel, and this also affects the objects which are in the environment.

The $\mathrm{P}$ colony executes a computation by synchronously applying the programs to the objects inside the cells and outside in the environment until a halting configuration is reached. The result of the computation is obtained as the vector of copies of certain "final" objects present in the environment after the system halts. 
In the following, after providing the formal definitions, we first give a short overview of results on the computational completeness of the different $\mathrm{P}$ colony variants. Then we present new results about two types of systems: first about the simplest possible P colonies, those which only have one object inside every cell, and then about a new type called P colonies with senders and consumers, which have special rules for insertion-deletion. We show that both kinds of these very simple devices are able to compute any recursively enumerable set of vectors of non-negative integers.

\section{Preliminaries}

Let $V$ be an alphabet, let $V^{*}$ be the set of all words over $V$, and let $\varepsilon$ denote the empty word. We denote the number of occurrences of a symbol $a \in V$ in $w$ by $|w| a$. The set of non-negative integers is denoted by $\mathbb{N}$.

A multiset over an arbitrary (not necessarily finite) set $V$ is a mapping $M: V \rightarrow \mathbb{N}$ which assigns to each object $a \in V$ its multiplicity $M(a)$ in $M$. The support of $M$ is the set $\operatorname{supp}(M)=\{a \mid M(a) \geq 1\}$. If $V$ is a finite set, then $M$ is called a finite multiset. A multiset $M$ is empty if its support is empty, $\operatorname{supp}(M)=\emptyset$. We will represent a finite multiset $M$ over $V$ by a string $w$ over the alphabet $V$ with $|w|_{a}=M(a), a \in V$, and $\varepsilon$ will represent the empty multiset.

We will also need the notion of a register machine which consists of a finite number of registers each of which can hold an arbitrarily large non-negative integer (we say that the register is empty if it holds zero), and a set of labeled instructions which specify how the numbers stored in the registers can be changed.

Formally, a register machine is a construct $M=\left(m, H, l_{0}, l_{h}, R\right)$, where $m$ is the number of registers, $H$ is the set of instruction labels, $l_{0}$ is the start label, $l_{h}$ is the halting label, and $R$ is the set of instructions. Each label from $H$ labels only one instruction from $R$. There are several types of instructions which can be used. For $l_{i}, l_{j}, l_{k} \in H$ and $r \in\{1, \ldots, m\}$ we have

- $l_{i}:\left(A D D(r), l_{j}, l_{k}\right)$ - nondeterministic add: Add one to register $r$ and then go to one of the instructions with labels $l_{j}$ or $l_{k}$, non-deterministically chosen.

- $l_{i}:\left(S U B(r), l_{j}, l_{k}\right)$ - subtract: If register $r$ is non-empty, then subtract one from it and go to the instruction with label $l_{j}$, if the value of register $r$ is zero, go to instruction $l_{k}$.

- $l_{h}: H A L T$ - halt: Stop the machine.

A register machine $M$ computes a set $N(M)$ of numbers in the following way: It starts with empty registers by executing the instruction with label $l_{0}$ and proceeds by applying instructions as indicated by the labels (and made possible by the contents of the registers). If the halt instruction is reached, then the number stored at that time in register 1 is said to be computed by $M$. Because of the non-determinism in choosing the continuation of the computation in the case of $A D D$ instructions, $N(M)$ can be an infinite set.

It is known (see, e.g., [6]) that in this way we can compute all sets of numbers which are Turing computable.

If a set of output registers $i_{1}, \ldots, i_{r}, 1 \leq r \leq m, i_{j} \in\{1, \ldots, m\}$ is specified, then $M$ computes a set of vectors of non-negative integers as follows. If the halt instruction is reached, then $\left(v_{1}, \ldots, v_{r}\right)$, where $v_{k}$ is the number stored in register $i_{k}, 1 \leq k \leq r$, is the vector of numbers computed by $M$, i.e., the result of that computation.

Now we recall the definition of a $\mathrm{P}$ colony from [5]. A $P$ colony is a construct $\Pi=\left(V, e, F, C_{1}, \ldots, C_{n}\right)$, $n \geq 1$, where $V$ is an alphabet (its elements are called objects). There are two kinds of distinguished objects: $e \in V$ (the environmental object), and the objects in $F \subseteq V$ (the set of final objects). The cells of the colony are denoted by $C_{1}, \ldots, C_{n}$. Each cell is a pair $C_{i}=\left(O_{i}, P_{i}\right)$, where $O_{i}$ is a multiset over $\{e\}$ 
having the same cardinality called capacity (here we only consider $\left|O_{i}\right| \in\{1,2\}$ ) for all $i, 1 \leq i \leq n$ (the initial state of the cell), and $P_{i}$ is a finite set of programs. Each program consists of rules of the following forms:

- $a \rightarrow b$ (internal point mutation), specifying that an object $a \in V$ inside the cell is changed to $b \in V$.

- $c \longrightarrow d$ (one object exchange with the environment), specifying that if $c \in V$ is contained inside the cell and $d \in V$ is present in the environment, then $c$ is sent out of the cell while $d$ is brought inside.

- $c \longrightarrow d / c \longrightarrow d^{\prime}$ (checking rule for one object exchange with the environment), specifying that if $c \in V$ is inside the cell then it is exchanged with $d \in V$ from the environment, or if there is no $d$ outside but $d^{\prime} \in V$ is present, then $c$ is exchanged with $d^{\prime}$.

- $c \longrightarrow d / c \rightarrow d^{\prime}$ (checking rule for one object exchange with the environment or internal point mutation), specifying that if the exchange of $c \in V$ inside and $d \in V$ outside is not possible, then $c$ is changed to $d^{\prime} \in V$.

The programs contain one rule for each element of $O_{i}$, thus, the number of rules of a program coincides with the cardinality of $O_{i}, 1 \leq i \leq n$.

In addition, $\mathrm{P}$ colonies with capacity of two may have programs of the form

- $\langle a, i n ; b c \rightarrow d\rangle$ with $a, b, c, d \in V$ (deletion programs), specifying that if $b c$ is present inside the cell and $a$ is present in the environment, then the objects inside are changed to $d$ and $a$ is brought in ( $a$ is "deleted" from the environment).

- $\langle a$, out $; b \rightarrow c d\rangle$ with $a, b, c, d \in V$ (insertion programs), specifying that if $a b$ is inside the cell, then $a$ is sent out ( $a$ is "inserted" into the environment) and $b$ is changed to $c d$.

The programs of the cells are used in the non-deterministic maximally parallel manner: in each time unit, each cell which is able to use one of its programs should use one. The use of a program means the application of the rule(s) of the program to the object(s) in the cell.

This way, transitions among the configurations of the colony are obtained. A sequence of transitions is a computation which is halting if it reaches a configuration where no cell can use any program. The result of a halting computation is obtained from the number of copies of objects from $F$ present in the environment in the halting configuration. Because of the non-determinism in choosing the programs, several computations can be obtained from a given initial configuration, hence with a $\mathrm{P}$ colony $\Pi$ we can associate a set of vectors of non-negative integers computed by all possible halting computations of $\Pi$.

Initially, the environment contains arbitrarily many copies of the environmental object $e$, and the cells also contain one or two copies of $e$ inside, depending on the capacity of the P colony.

For a $\mathrm{P}$ colony $\Pi=\left(V, e, F, C_{1}, \ldots, C_{n}\right)$ as above, a configuration can be formally written as an $(n+1)$ tuple

$$
\left(w_{1}, \ldots, w_{n} ; w_{E}\right)
$$

where $w_{i} \in V^{*}$ represents the multiset of objects from cell $C_{i}, 1 \leq i \leq n$, and $w_{E} \in(V-\{e\})^{*}$ represents the multiset of objects from the environment different from the environmental object $e$. The initial configuration is $\left(e^{i}, \ldots, e^{i} ; \varepsilon\right)$ where $i \in\{1,2\}$ is the capacity of the cells.

A transition from a configuration to another is denoted as

$$
\left(w_{1}, \ldots, w_{n} ; w_{E}\right) \Rightarrow\left(w_{1}^{\prime}, \ldots, w_{n}^{\prime} ; w_{E}^{\prime}\right)
$$

where $w_{E}^{\prime}$ and each $w_{i}^{\prime}$ is obtained from $w_{i}, 1 \leq i \leq n$, and $w_{E}$ by executing one of the programs of $P_{i}$. 
The set of vectors in $\mathbb{N}^{m}, m=|F|, F=\left\{o_{1}, \ldots, o_{m}\right\}$, computed by a $\mathrm{P}$ colony $\Pi$ is defined as

$$
N(\Pi)=\left\{\left(\left|v_{E}\right|_{o_{1}}, \ldots,\left|v_{E}\right|_{o_{m}}\right) \mid\left(e^{i}, \ldots, e^{i} ; \varepsilon\right) \Rightarrow^{*}\left(v_{1}, \ldots, v_{n}, v_{E}\right)\right\}
$$

where $\left(e^{i}, \ldots, e^{i}, \varepsilon\right), i \in\{1,2\}$, is the initial configuration, $\left(v_{1}, \ldots, v_{n}, v_{E}\right)$ is a halting configuration, and $\Rightarrow^{*}$ denotes the reflexive and transitive closure of $\Rightarrow$.

Let us denote by $\operatorname{PCOL}(i, j, k$, check $)$ and $\operatorname{PCOL}(i, j, k$, no-check $)$ the classes of sets of vectors generated by $\mathrm{P}$ colonies with at most $j \geq 1$ cells of capacity $i \in\{1,2\}$, having at most $k \geq 1$ programs associated to a cell which contain or do not contain checking rules, respectively. If a numerical parameter is unbounded, we denote it by a $*$.

$\mathrm{P}$ colonies can simulate register machines with a rather limited number of programs per cell. In [3], it was shown that

$$
\operatorname{PCOL}(2, *, 4, \operatorname{check})=\operatorname{PCOL}(3, *, 3, \text { check })=\mathbb{N} R E
$$

where $\mathbb{N} R E$ denotes the class of recursively enumerable sets of integer vectors. Even one cell is enough, if it may have an arbitrarily large number of programs, that is,

$$
\operatorname{PCOL}(2,1, *, \text { check })=\mathbb{N} R E .
$$

Similar results were also obtained without the use of checking rules. In this case we have

$$
\operatorname{PCOL}(2, *, 8, \text { no-check })=\operatorname{PCOL}(3, *, 7, \text { no-check })=\mathbb{N} R E .
$$

\section{P colonies with one object}

In [1] it was shown that if checking rules are allowed to be used, then all recursively enumerable sets of vectors can even be generated by $\mathrm{P}$ colonies with capacity one, that is,

$$
\operatorname{PCOL}(1,4, *, \operatorname{check})=\mathbb{N} R E .
$$

In the following we show that $\mathrm{P}$ colonies with six components generate all vectors even if checking rules are not used.

Theorem 1. $\operatorname{PCOL}(1,6, *$, no-check $)=\mathbb{N} R E$.

Proof. We construct a $\mathrm{P}$ colony simulating the computations of a register machine. Let us consider an $m$ register machine $M=\left(m, H, l_{0}, l_{h}, P\right)$ and represent the content of the register $i$ by the number of copies of a specific object $a_{i}$ in the environment. We construct the $\mathrm{P}$ colony $\Pi=\left(V, e, F, C_{1}, \ldots, C_{6}\right)$ with:

$$
\begin{aligned}
V= & \left\{e, l_{i}, l_{i}^{\prime}, l_{i}^{\prime \prime}, \bar{l}_{i}, K_{i}, L_{i}, L_{i}^{\prime}, L_{i}^{\prime \prime}, L_{i}^{\prime \prime \prime}, E_{i}, F_{i}, \$_{i} \mid \text { for each } l_{i} \in H\right\} \cup \\
& \left\{a_{i}, a_{i, j}|1 \leq i \leq m, 1 \leq j \leq| H \mid\right\} \cup\left\{D, D^{\prime}, T\right\}, \\
F= & \left\{a_{i} \mid \text { register } i \text { is an output register }\right\}, \text { and } \\
C_{i}= & \left(e, P_{i}\right), \text { for } 1 \leq i \leq 6 .
\end{aligned}
$$

Because initially there are only copies of $e$ in the environment and inside the cells, we have to initialize the simulation of the computation of $M$ by generating the initial the label $l_{0}$, and an arbitrary number of $l_{i}^{\prime}, l_{i}^{\prime \prime}$ for all $l_{i} \in H$. These symbols are generated by $C_{1}$ and $C_{2}$ with the following programs:

$$
\begin{aligned}
P_{1} \supset & \left\{\left\langle e \rightarrow l_{r}^{\prime}\right\rangle,\left\langle l_{r}^{\prime} \longrightarrow e\right\rangle,\left\langle e \rightarrow l_{r}^{\prime \prime}\right\rangle,\left\langle l_{r}^{\prime \prime} \longrightarrow e\right\rangle \mid l_{r} \in H\right\} \cup \\
& \left\{\left\langle e \longrightarrow D^{\prime}\right\rangle,\left\langle D^{\prime} \rightarrow l_{0}\right\rangle,\left\langle l_{0} \longrightarrow D\right\rangle\right\}, \\
P_{2} \supset & \left\{\left\langle e \rightarrow D^{\prime}\right\rangle,\left\langle D^{\prime} \rightarrow D^{\prime}\right\rangle,\left\langle D^{\prime} \longrightarrow l_{1}^{\prime}\right\rangle,\left\langle l_{1}^{\prime} \rightarrow D\right\rangle,\left\langle D \longrightarrow l_{1}^{\prime \prime}\right\rangle\right\} .
\end{aligned}
$$


With these programs, from the configuration $(e, e, e, e, e, e ; \varepsilon)$, we obtain $\left(D, l_{1}^{\prime \prime}, e, e, e, e ; l_{0} w\right)$ where the environment contains the label of the initial instruction, $l_{0}$, and $w$, a multiset of primed and double primed instruction labels.

To simulate the instruction $l_{i}:\left(A D D(r), l_{j}, l_{k}\right)$, cells $C_{1}$ and $C_{3}$ cooperate to add one copy of object $a_{r}$ and object $l_{j}$ or $l_{k}$ to the environment.

\begin{tabular}{llllllll}
$P_{1}$ & \multicolumn{7}{c}{$P_{3}$} \\
\hline$i_{1}:$ & $\left\langle D \longrightarrow a_{r, i}\right\rangle$ & $i_{6}:$ & $\left\langle K_{k} \rightarrow l_{k}\right\rangle$ & $i_{1}:$ & $\left\langle e \longrightarrow l_{i}\right\rangle$ & $i_{6}:$ & $\left\langle l_{i}^{\prime} \rightarrow K_{k}\right\rangle$ \\
$i_{2}:$ & $\left\langle a_{r, i} \rightarrow a_{r}\right\rangle$ & $i_{7}:$ & $\left\langle l_{j} \longrightarrow D\right\rangle$ & $i_{2}:$ & $\left\langle l_{i} \rightarrow a_{r, i}\right\rangle$ & $i_{7}:$ & $\left\langle K_{j} \longrightarrow e\right\rangle$ \\
$i_{3}:$ & $\left\langle a_{r} \longrightarrow K_{j}\right\rangle$ & $i_{8}:$ & $\left\langle l_{k} \longrightarrow D\right\rangle$ & $i_{3}:$ & $\left\langle a_{r, i} \longrightarrow l_{i}^{\prime}\right\rangle$ & $i_{8}:$ & $\left\langle K_{k} \longrightarrow e\right\rangle$ \\
$i_{4}:$ & $\left\langle a_{r} \longrightarrow K_{k}\right\rangle$ & & & $i_{4}:$ & $\left\langle a_{r, i} \rightarrow t\right\rangle$ & $i_{9}:$ & $\langle t \rightarrow t\rangle$ \\
$i_{5}:$ & $\left\langle K_{j} \rightarrow l_{j}\right\rangle$ & & & $i_{5}:$ & $\left\langle l_{i}^{\prime} \rightarrow K_{j}\right\rangle$ & &
\end{tabular}

It is not difficult to follow how the interplay of these two cells produce the configuration $\left(D, l_{1}^{\prime \prime}, e, e, e, e ; l_{j} a_{r} w^{\prime}\right)$ or $\left(D, l_{1}^{\prime \prime}, e, e, e, e ; l_{k} a_{r} w^{\prime}\right)$ from a configuration $\left(D, l_{1}^{\prime \prime}, e, e, e, e ; l_{i} w\right)$ where $w, w^{\prime}$ are multisets of $l_{i}^{\prime}, l_{i}^{\prime \prime}$ for $l_{i} \in H$ and $a_{r}, 1 \leq r \leq m$. If there is no $l_{i}^{\prime}$ present in the environment when the program $i_{3}$ of cell $C_{3}$ should be used, then the programs $i_{4}$ and $i_{9}$ do not allow the halting of the computation.

For each subtract instruction $l_{f}:\left(S U B(r), l_{g}, l_{n}\right)$ there are the following programs in $P_{1}, P_{4}, P_{5}$ and in $P_{6}$ :

\begin{tabular}{|c|c|c|c|c|}
\hline$P_{1}$ & $P_{4}$ & & $P_{5}$ & $P_{6}$ \\
\hline$f_{1}:\left\langle D \leftrightarrow L_{f}\right\rangle$ & $f_{1}:\left\langle e \longrightarrow l_{f}\right\rangle$ & $f_{9}: \quad\left\langle L_{f} \rightarrow t\right\rangle$ & $f_{1}:\left\langle e \leftrightarrow L_{f}^{\prime}\right\rangle$ & $f_{1}:\left\langle e \leftrightarrow L_{f}^{\prime \prime}\right\rangle$ \\
\hline$f_{2}:\left\langle L_{f} \rightarrow E_{f}\right\rangle$ & $f_{2}:\left\langle l_{f} \rightarrow L_{f}\right\rangle$ & $f_{10}:\left\langle L_{f}^{\prime} \rightarrow t\right\rangle$ & $f_{2}:\left\langle L_{f}^{\prime} \rightarrow l_{f}^{\prime}\right\rangle$ & $\left\langle L_{f}^{\prime \prime} \rightarrow l_{f}^{\prime}\right\rangle$ \\
\hline$f_{3}: \quad\left\langle E_{f} \rightarrow F_{f}\right\rangle$ & $f_{3}:\left\langle L_{f} \leftrightarrow l_{f}^{\prime}\right\rangle$ & $f_{11}:\langle t \rightarrow t\rangle$ & $f_{3}: \quad\left\langle l_{f}^{\prime} \leftrightarrow a_{r}\right\rangle$ & $f_{3}:\left\langle l_{f}^{\prime} \longrightarrow \$_{f}\right\rangle$ \\
\hline$f_{4}: \quad\left\langle F_{f} \rightarrow \$_{f}\right\rangle$ & $f_{4}:\left\langle l_{f}^{\prime} \rightarrow L_{f}^{\prime}\right\rangle$ & & $f_{4}:\left\langle l_{f}^{\prime} \leftrightarrow \$_{f}\right\rangle$ & $f_{4}:\left\langle \$_{f} \rightarrow l_{g}\right\rangle$ \\
\hline$f_{5}: \quad\left\langle \$_{f} \leftrightarrow D\right\rangle$ & $f_{5}:\left\langle L_{f}^{\prime} \longrightarrow l_{f}^{\prime \prime}\right\rangle$ & & $f_{5}: \quad\left\langle \$_{f} \rightarrow \bar{l}_{n}\right\rangle$ & $f_{5}:\left\langle l_{g} \leftrightarrow e\right\rangle$ \\
\hline & $f_{6}:\left\langle l_{f}^{\prime \prime} \rightarrow L_{f}^{\prime \prime \prime}\right\rangle$ & & $f_{6}:\left\langle a_{r} \rightarrow e\right\rangle$ & $f_{6}:\left\langle l_{f}^{\prime} \longrightarrow \bar{l}_{n}\right\rangle$ \\
\hline & $f_{7}:\left\langle L_{f}^{\prime \prime \prime} \rightarrow L_{f}^{\prime \prime}\right\rangle$ & & $f_{7}:\left\langle\bar{l}_{n} \leftrightarrow e\right\rangle$ & $f_{7}:\left\langle\vec{l}_{n} \rightarrow l_{n}\right\rangle$ \\
\hline & $f_{8}:\left\langle L_{f}^{\prime \prime} \longrightarrow e\right\rangle$ & & & $f_{8}:\left\langle l_{n} \longrightarrow e\right\rangle$ \\
\hline
\end{tabular}

In the following table we show how a subtract instruction can be simulated by the programs above. Since $C_{2}$ and $C_{3}$ cannot apply any of their rules in any step of the following simulation, we omit them from the table. The multiset of objects in the environment is denoted by [...], and for now we assume that it always contains a sufficient amount of $l_{i}^{\prime}, l_{i}^{\prime \prime}$ objects for any $l_{i} \in H$.

First we consider the case when there is at least one object $a_{r}$ in the environment, that is, if the 
simulation starts in a configuration $\left(D, l_{1}^{\prime \prime}, e, e, e, e ; l_{f} a_{r}[\ldots]\right)$.

\begin{tabular}{|c|rrrrr|rrrr|}
\hline & \multicolumn{7}{|c|}{ configuration of $\Pi$} & \multicolumn{5}{c|}{ programs to be applied } \\
& $C_{1}$ & $C_{4}$ & $C_{5}$ & $C_{6}$ & $E n v$ & $P_{1}$ & $P_{4}$ & $P_{5}$ & $P_{6}$ \\
\hline 1. & $D$ & $e$ & $e$ & $e$ & $l_{f} a_{r}[\ldots]$ & - & $f_{1}$ & - & - \\
2. & $D$ & $l_{f}$ & $e$ & $e$ & $a_{r}[\ldots]$ & - & $f_{2}$ & - & - \\
3. & $D$ & $L_{f}$ & $e$ & $e$ & $a_{r}[\ldots]$ & - & $f_{3}$ & - & - \\
4. & $D$ & $l_{f}^{\prime}$ & $e$ & $e$ & $L_{f} a_{r}[\ldots]$ & $f_{1}$ & $f_{4}$ & - & - \\
5. & $L_{f}$ & $L_{f}^{\prime}$ & $e$ & $e$ & $D a_{r}[\ldots]$ & $f_{2}$ & $f_{5}$ & - & - \\
6. & $E_{f}$ & $l_{f}^{\prime \prime}$ & $e$ & $e$ & $L_{f}^{\prime} D a_{r}[\ldots]$ & $f_{3}$ & $f_{6}$ & $f_{1}$ & - \\
7. & $F_{f}$ & $L_{f}^{\prime \prime}$ & $L_{f}^{\prime}$ & $e$ & $D a_{r}[\ldots]$ & $f_{4}$ & $f_{7}$ & $f_{2}$ & - \\
8. & $\$$ & $L_{f}^{\prime \prime}$ & $l_{f}^{\prime}$ & $e$ & $D a_{r}[\ldots]$ & $f_{5}$ & $f_{8}$ & $f_{3}$ & - \\
9. & $D$ & $e$ & $a_{r}$ & $e$ & $\$_{f} L_{f}^{\prime \prime}[\ldots]$ & - & - & $f_{6}$ & $f_{1}$ \\
10. & $D$ & $e$ & $e$ & $L_{f}^{\prime \prime}$ & $\$_{f}[\ldots]$ & - & - & - & $f_{2}$ \\
11. & $D$ & $e$ & $e$ & $l_{f}^{\prime}$ & $\$_{f}[\ldots]$ & - & - & - & $f_{3}$ \\
12. & $D$ & $e$ & $e$ & $\$$ & {$[\ldots]$} & - & - & - & $f_{4}$ \\
13. & $D$ & $e$ & $e$ & $l_{g}$ & {$[\ldots]$} & - & - & - & $f_{5}$ \\
\hline 14. & $D$ & $e$ & $e$ & $e$ & $l_{g}[\ldots]$ & - & $g_{1}$ & - & - \\
\hline
\end{tabular}

In 13 steps, from a configuration $\left(D, l_{1}^{\prime \prime}, e, e, e, e ; l_{f} a_{r}[\ldots]\right)$ we obtain $\left(D, l_{1}^{\prime \prime}, e, e, e, e ; l_{g}[\ldots]\right)$ where $l_{g}$ is the label of the instruction which should follow the successful decrease of the value of the nonempty register $r$, and the environment contains a multiset of objects $l_{i}^{\prime}, l_{i}^{\prime \prime}$ for $l_{i} \in H$.

Now we consider the case when register $r$, which is the register to be decremented, stores zero, that is, if the simulation starts in a configuration $\left(D, l_{1}^{\prime \prime}, e, e, e, e ; l_{f}[\ldots]\right)$ where the environment does not contain any object $a_{r}$.

\begin{tabular}{|c|ccccr|cccc|}
\hline & \multicolumn{7}{|c|}{ configuration of $\Pi$} & \multicolumn{5}{|c|}{ programs to be applied } \\
\hline & $C_{1}$ & $C_{4}$ & $C_{5}$ & $C_{6}$ & $E n v$ & $P_{1}$ & $P_{4}$ & $P_{5}$ & $P_{6}$ \\
\hline 1. & $D$ & $e$ & $e$ & $e$ & $l_{f}[\ldots]$ & - & $f_{1}$ & - & - \\
2. & $D$ & $l_{f}$ & $e$ & $e$ & {$[\ldots]$} & - & $f_{2}$ & - & - \\
3. & $D$ & $L_{f}$ & $e$ & $e$ & {$[\ldots]$} & - & $f_{3}$ & - & - \\
4. & $D$ & $l_{f}^{\prime}$ & $e$ & $e$ & $L_{f}[\ldots]$ & $f_{1}$ & $f_{4}$ & - & - \\
5. & $L_{f}$ & $L_{f}^{\prime}$ & $e$ & $e$ & $D[\ldots]$ & $f_{2}$ & $f_{5}$ & - & - \\
6. & $E_{f}$ & $l_{f}^{\prime \prime}$ & $e$ & $e$ & $L_{f}^{\prime} D[\ldots]$ & $f_{3}$ & $f_{6}$ & $f_{1}$ & - \\
$7 \cdot$ & $F_{f}$ & $L_{f}^{\prime \prime \prime}$ & $L_{f}^{\prime}$ & $e$ & $D[\ldots]$ & $f_{4}$ & $f_{7}$ & $f_{2}$ & - \\
8. & $\$$ & $L_{f}^{\prime \prime}$ & $l_{f}^{\prime}$ & $e$ & $D[\ldots]$ & $f_{5}$ & $f_{8}$ & - & - \\
9. & $D$ & $e$ & $l_{f}^{\prime}$ & $e$ & $\$_{f} L_{f}^{\prime \prime}[\ldots]$ & - & - & $f_{4}$ & $f_{1}$ \\
10. & $D$ & $e$ & $\$ f$ & $L_{f}^{\prime \prime}$ & {$[\ldots]$} & - & - & $f_{5}$ & $f_{2}$ \\
11. & $D$ & $e$ & $\overline{l_{n}}$ & $l_{f}^{\prime}$ & {$[\ldots]$} & - & - & $f_{7}$ & - \\
12. & $D$ & $e$ & $e$ & $l_{f}^{\prime}$ & $\bar{l}_{n}[\ldots]$ & - & - & - & $f_{6}$ \\
13. & $D$ & $e$ & $e$ & $\bar{l}_{n}$ & {$[\ldots]$} & - & - & - & $f_{7}$ \\
14. & $D$ & $e$ & $e$ & $l_{n}$ & {$[\ldots]$} & - & - & - & $f_{8}$ \\
\hline 15. & $D$ & $e$ & $e$ & $e$ & $l_{n}[\ldots]$ & - & $n_{1}$ & - & - \\
\hline
\end{tabular}

Similarly to the previous case, in 14 steps we obtain a configuration $\left(D, l_{1}^{\prime \prime}, e, e, e, e ; l_{n}[\ldots]\right)$ where $l_{n}$ is the label of the instruction which should follow $l_{f}$ if register $r$ is empty, that is, if the decrease of its value is not possible.

Consider now what happens if there is an insufficient amount of objects $l_{i}^{\prime}, l_{i}^{\prime \prime}$ for $l_{i} \in H$ is present in the environment. Notice that such symbols are needed in step 3 and 5 by cell $C_{4}$. If there is no more 
available (not enough of them were produced in the initial phase by $C_{1}$ and $C_{2}$ ), then the programs $f_{9}$, $f_{10}$, and $f_{11}$ do not allow the halting of the computation.

From these considerations we can see that after the initialization phase, all instructions of the register machine $M$ can be simulated by the $\mathrm{P}$ colony. If the label of the halt instruction, $l_{h}$ is produced, the computation halts since there is no program for processing the object $l_{h}$. The reader can immediately see that $\Pi$ computes the same set of vectors as $M$.

\section{P colonies with senders and consumers}

Now we continue with the investigation of two object $\mathrm{P}$ colonies with insertion-deletion programs. It is not too difficult to see that if we allow a cell to contain both types of programs, then we can simulate the other types of programs in two steps, thus, it is more interesting to consider P colonies having cells which contain either insertion or deletion programs, but not both types at the same time. We call these systems P colonies with senders and consumers. A sender is a cell with only insertion programs, a consumer is a cell with only deletion programs.

Let us denote by $\operatorname{PCOL}(i, j, \mathrm{~s}-\mathrm{c})$ the class of sets of numbers generated by $\mathrm{P}$ colonies with senders and consumers having at most $i \geq 1$ cells with at most $j \geq 1$ program each.

Example 2. (a) A P colony with one sender cell can generate the Parikh set of a regular language $L \subseteq T^{*}$. Let $G=(N, T, P, S)$ be a regular grammar such that $L(G)=L$.

For generating the Parikh vectors of the words in $L$, we use, for each $S \rightarrow a B$ of $P$, the programs $\langle e$, out $; e \rightarrow e S\rangle,\langle e$, out $; S \rightarrow a B\rangle$ and then $\langle x$, out $; A \rightarrow a B\rangle, x \in T$ for every $A \rightarrow a B$ in $P$. Finally, for every rule of the form $A \rightarrow$ we need $\langle x$, out $; A \rightarrow a F\rangle, x \in T,\langle a$, out $; F \rightarrow F F\rangle$, where $F \notin T \cup N$.

(b) A P colony with one consumer cell can "consume" the Parikh set of a regular language $L$. To see this, let $M=\left(Q, T, \delta, q_{0}, F\right)$ be a deterministic finite automaton such that $L(M)=L$.

We need the program $\left\langle e\right.$,in;ee $\left.\rightarrow q_{0}\right\rangle$, and to every transition $\delta\left(q_{i}, a\right)=q_{j}$ in $M$, we introduce $\left\langle a\right.$, in $\left.; x q_{i} \rightarrow q_{j}\right\rangle, x \in T \cup\{e\}$. If $q_{j} \in F$ in $\delta\left(q_{i}, a\right)=q_{j}$ we have to add the programs $\left\langle a, i n ; x q_{i} \rightarrow F\right\rangle, x \in T$, where $F \notin Q \cup T$.

Now we show that three cells, one sender and two consumers are sufficient to generate all recursively enumerable sets of integer vectors.

Theorem 3. $\operatorname{PCOL}(3, *, s-c)=\mathbb{N} R E$.

Proof. Consider an $m$-register machine $M=\left(m, H, l_{0}, l_{h}, P\right), m \geq 1$. We simulate $M$ by representing the content of the register $i$ by the number of copies of a specific object $a_{i}$ in the environment. We construct the $\mathrm{P}$ colony $\Pi=\left(V, e, F, C_{1}, C_{2}, C_{3}\right)$ with:

$$
\begin{aligned}
V & =\left\{e, l, l^{\prime}, l^{\prime \prime}, l^{\prime \prime \prime}, l^{i v}, l^{v}, \bar{l}, \overline{\bar{l}} \mid l \in H\right\} \cup\left\{a_{i} \mid 1 \leq i \leq m\right\} \cup\left\{K, T_{1}, T_{2}, T_{3}, T_{4}, T_{5}\right\} \\
F & =\left\{a_{i} \mid \text { register } i \text { is an output register }\right\}, \text { and } \\
C_{i} & =\left(e e, P_{i}\right) \text { for } 1 \leq i \leq 3 .
\end{aligned}
$$

The $\mathrm{P}$ colony $\Pi$ starts its computation in the initial configuration $(e e, e e, e e ; \varepsilon)$. We initialize the computation by generating the initial label $l_{0}$ with a program from $P_{1}$, $\left\langle e\right.$, out; $\left.e \rightarrow l_{0} l_{0}\right\rangle \in P_{1}$ obtaining $\left(l_{0} l_{0}, e e, e e ; \varepsilon\right)$.

The simulation of an instruction with label $l_{i}$ starts from a configuration $\left(l_{i} l_{i}, e e, e e ; w\right)$ where $w \in V^{*}$, the multiset of objects in the environment, represents the counter contents of $M$.

To simulate an $A D D$ instruction, we use the programs of $P_{1}$ and $P_{3}$. For each $l_{i}, l_{j}, l_{k} \in H$ with $l_{i}$ being 
the label of an instruction $l_{i}:\left(A D D(r), l_{j}, l_{k}\right)$, we have the following programs:

\begin{tabular}{clll}
$P_{1}$ & & $P_{3}$ & \\
\hline$i_{1}:$ & $\left\langle l_{i}\right.$, out $\left.; l_{i} \rightarrow a_{r} l_{j}\right\rangle$ & $i_{1}:$ & $\left\langle l_{i}\right.$, in $;$ ee $\left.\rightarrow T_{1}\right\rangle$ \\
$i_{2}:$ & $\left\langle l_{i}\right.$, out $\left.; l_{i} \rightarrow a_{r} l_{k}\right\rangle$ & $i_{2}:$ & $\left\langle e\right.$, in $\left.; l_{i} T_{1} \rightarrow e\right\rangle$ \\
$i_{3}:$ & $\left\langle a_{r}\right.$, out $\left.; l_{j} \rightarrow l_{j} l_{j}\right\rangle$ & $i_{3}:$ & $\left\langle l_{i}\right.$, in $\left.; \bar{l}_{i} T_{5} \rightarrow T_{1}\right\rangle$ \\
$i_{4}:$ & $\left\langle a_{r}\right.$, out $\left.; l_{k} \rightarrow l_{k} l_{k}\right\rangle$ & &
\end{tabular}

Using these programs, we obtain a sequence of configurations

$$
\left(l_{i} l_{i}, e e, e e ; w\right) \Rightarrow\left(a_{r} l, e e, e e ; l_{i} w\right) \Rightarrow\left(l l, e e, l_{i} T_{1} ; a_{r} w\right)
$$

where $l$ is the label of the next instruction, that is, we either have $\left(l_{j} l_{j}, e e, l_{i} T_{1} ; a_{r} w\right)$, or the configuration $\left(l_{k} l_{k}, e e, l_{i} T_{1} ; a_{r} w\right)$. The contents of cell $C_{3}, l_{i} T_{1}$, will change in the next step to $e e$ independently of the several ways of the continuation of the computation, as we shall see later.

The program labeled with $i_{3}$ is used if the instruction simulated before $l_{i}$ was a SUB instruction (see below). In this case, the configuration in which the simulation of $l_{i}$ starts is $\left(l_{i} l_{i}, e e, \bar{l}_{i} T_{4} ; \overline{\bar{l}}_{i} w\right)$ and we need the steps $\left(l_{i} l_{i}, e e, \bar{l}_{i} T_{4} ; \bar{l}_{i} w\right) \Rightarrow\left(a_{r} l, e e, \overline{\bar{l}}_{i} T_{5} ; l_{i} w\right) \Rightarrow\left(l l, e e, l_{i} T_{1} ; a_{r} w\right)$ and program $i_{3}$ to obtain the same configuration as before.

Now we show how to simulate a SUB instruction. For each $l_{j}, l_{k}, l_{l} \in H$ with $l_{j}$ being the label of an instruction $l_{j}:\left(S U B(r), l_{k}, l_{l}\right)$, and for all labels $l_{s} \in H$, we have the following programs.

\begin{tabular}{|c|c|c|c|c|}
\hline$P_{1}$ & $P_{2}$ & & $P_{3}$ & \\
\hline$j_{1}:\left\langle l_{j}\right.$, out $\left.; l_{j} \rightarrow l_{j}^{\prime} l_{j}^{\prime}\right\rangle$ & $j_{1}:$ & $\left\langle l_{j}\right.$, in;ee $\left.\rightarrow e\right\rangle$ & $j_{1}:$ & $\left\langle l_{j}^{\prime}\right.$, in;ee $\left.\rightarrow T_{1}\right\rangle$ \\
\hline$j_{2}:\left\langle l_{j}^{\prime}\right.$, out $\left.; l_{j}^{\prime} \rightarrow l_{j}^{\prime \prime} l_{j}^{\prime \prime}\right\rangle$ & $j_{2}:$ & $\left\langle a_{r},{\left.\text { in } ; e l_{j} \rightarrow e\right\rangle} \rightarrow\right.$ & $j_{2}:$ & $\left\langle e, i n ; l_{j}^{\prime} T_{1} \rightarrow T_{2}\right\rangle$ \\
\hline$j_{3}:\left\langle l_{j}^{\prime \prime}\right.$, out $\left.; l_{j}^{\prime \prime} \rightarrow l_{j}^{\prime \prime \prime} l_{j}^{i v}\right\rangle$ & $j_{3}:$ & $\left\langle l_{j}^{\prime \prime}\right.$, in$\left._{j} e l_{j} \rightarrow e\right\rangle$ & $j_{3}:$ & $\left\langle l_{j}^{\prime \prime}, i n ; e T_{2} \rightarrow T_{3}\right\rangle$ \\
\hline$j_{4}:\left\langle l_{j}^{\prime \prime \prime}\right.$, out $\left.; l_{j}^{i v} \rightarrow \bar{l}_{k} \bar{l}_{k}\right\rangle$ & $j_{4}:$ & $\left\langle l_{j}^{\prime \prime \prime}, i n ; a_{r} e \rightarrow e\right\rangle$ & $j_{4, s}:$ & $\left\langle\bar{l}_{s}, i n ; l_{j}^{\prime \prime} T_{3} \rightarrow T_{4}\right\rangle$ \\
\hline$j_{5}:\left\langle l_{j}^{i v}\right.$, out $\left.; l_{j}^{\prime \prime \prime \prime} \rightarrow \bar{l}_{l} \bar{l}_{l}\right\rangle$ & $j_{5}:$ & $\left\langle e, i n ; l_{j}^{\prime \prime \prime} e \rightarrow e\right\rangle$ & $j_{5, s}:$ & $\left\langle\bar{l}_{s}, i n ; e T_{2} \rightarrow T_{4}\right\rangle$ \\
\hline$j_{6}:\left\langle\overline{\bar{l}}_{\underline{k}}\right.$, out $\left.; \bar{l}_{\underline{k}} \rightarrow \overline{\bar{l}}_{k} \overline{\bar{l}}_{k}\right\rangle$ & $j_{6}:$ & $\left\langle l_{j}^{i v}, i n ; a_{r} e \rightarrow K\right\rangle$ & $j_{6, s}:$ & $\left\langle\overline{\bar{l}}, i n ; \bar{l}_{s} T_{4} \rightarrow T_{5}\right\rangle$ \\
\hline$j_{7}:\left\langle\overline{\bar{l}_{k}}\right.$, out $\left.; \overline{\bar{l}}_{k} \rightarrow l_{k} l_{k}\right\rangle$ & $j_{7}:$ & $\left\langle e, i n ; l_{j}^{i v} K \rightarrow K\right\rangle$ & $j_{7, s}:$ & $\left\langle e, i n ; \overline{\bar{l}}_{s} T_{5} \rightarrow e\right\rangle$ \\
\hline$j_{8}:\left\langle\bar{l}_{l}\right.$, out $\left.; \bar{l}_{l} \rightarrow \overline{\bar{l}}_{l} \overline{\bar{l}}_{l}\right\rangle$ & $j_{8}:$ & $\langle e, i n ; e K \rightarrow K\rangle$ & & \\
\hline$j_{9}:\left\langle\overline{\bar{l}}_{l}\right.$, out $\left.; \overline{\bar{l}}_{k} \rightarrow l_{l} l_{l}\right\rangle$ & $j_{9}:$ & $\left\langle l_{j}^{\prime \prime \prime}, i n ; l_{j}^{\prime \prime} e \rightarrow K\right\rangle$ & & \\
\hline & $j_{10}:$ & $\left\langle e, i n ; l_{j}^{\prime \prime \prime} K \rightarrow K\right\rangle$ & & \\
\hline & $j_{11}:$ & $\left\langle l_{j}^{i v}, i n ; l_{j}^{\prime \prime} e \rightarrow e\right\rangle$ & & \\
\hline & $j_{12}:$ & $\left\langle e, i n ; l_{j}^{i v} e \rightarrow e\right\rangle$ & & \\
\hline
\end{tabular}

In the following tables we show how the programs above simulate the execution of the instruction $l_{j}$ : $\left(S U B(r), l_{k}, l_{l}\right)$. To save space, we use the sign " " to separate the different possible multisets which might appear in the same row of the table.

First we consider the case when register $r$ is not empty, that is, when there is at least one object $a_{r}$ present in the environment. We see that starting with a configuration where $C_{1}$ contains the objects $l_{j} l_{j}$ and the environment contains $a_{r}$, in six steps we obtain a configuration where the object $a_{r}$ is removed from the environment, and $C_{1}$ either contains the label of the next instruction $l_{k}$, or because of the presence of program $j_{8}$, in $P_{2}$, the computation will never be able to halt. 


\begin{tabular}{|c|cccc|ccc|}
\hline & \multicolumn{5}{|c|}{ configuration of $\Pi$ programs to be applied } \\
& $C_{1}$ & $C_{2}$ & $C_{3}$ & $E n v$ & $P_{1}$ & $P_{2}$ & $P_{3}$ \\
\hline 1. & $l_{j} l_{j}$ & $e e$ & $?$ & $a_{r} w^{\prime}$ & $j_{1}$ & - & $?$ \\
2. & $l_{j}^{\prime} l_{j}^{\prime}$ & $e e$ & $?$ & $l_{j} a_{r} w^{\prime \prime}$ & $j_{2}$ & $j_{1}$ & $?$ \\
$3 \cdot$ & $l_{j}^{\prime \prime} l_{j}^{\prime \prime}$ & $l_{j} e$ & $e e$ & $l_{j}^{\prime} a_{r} w$ & $j_{3}$ & $j_{2}$ & $j_{1}$ \\
$4 \cdot$ & $l_{j}^{\prime \prime \prime} l_{j}^{i v}$ & $a_{r} e$ & $l_{j}^{\prime} T_{1}$ & $l_{j}^{\prime \prime} w$ & $j_{4} / j_{5}$ & - & $j_{2}$ \\
$5 \cdot$ & $\bar{l}_{k} \bar{l}_{k} / \bar{l}_{l} \bar{l}_{l}$ & $a_{r} e$ & $e T_{2}$ & $\left(l_{j}^{\prime \prime \prime} / l_{j}^{i v}\right) l_{j}^{\prime \prime} w$ & $j_{6} / j_{8}$ & $j_{4} / j_{6}$ & $j_{3}$ \\
6. & $\overline{\bar{l}}_{k} \overline{\bar{l}}_{k} / \overline{\bar{l}}_{l} \overline{\bar{l}}_{l}$ & $l_{j}^{\prime \prime \prime} e / l_{j}^{i v} K$ & $l_{j}^{\prime \prime} T_{3}$ & $\left(\bar{l}_{k} / \bar{l}_{l}\right) w$ & $j_{7} / j_{9}$ & $j_{5} / j_{7}$ & $j_{4, k} / j_{4, l}$ \\
\hline & $l_{k} l_{k} / l_{l} l_{l}$ & $e e / e K$ & $\left(\bar{l}_{k} / \bar{l}_{l}\right) T_{4}$ & $\left(\overline{\bar{l}}_{k} / \overline{\bar{l}}_{l}\right) w$ & $k_{1} / l_{1}$ & $-/ j_{8}$ & $j_{6, k} / j_{6, l}$ \\
$7 \cdot$ & $l_{k}^{\prime} l_{k}^{\prime} / l_{l}^{\prime} l_{l}^{\prime}$ & $e e / e K$ & $\left(\overline{\bar{l}}_{k} / \overline{\bar{l}}_{l}\right) T_{5}$ & $\left(l_{k} / l_{l}\right) w$ & $k_{2} / l_{2}$ & $k_{1} / j_{8}$ & $j_{7, k} / j_{7, l}$ \\
9. & $l_{k}^{\prime \prime} l_{k}^{\prime \prime} / l_{l}^{\prime \prime} l_{l}^{\prime \prime}$ & $\left(l_{k} / l_{l}\right) e / e K$ & $e e$ & $\left(l_{k}^{\prime} / l_{l}^{\prime}\right) w$ & $k_{3} / l_{3}$ & $k_{2} / j_{8}$ & $j_{1}$ \\
\hline
\end{tabular}

Now we show the simulation of the $l_{j}:\left(S U B(r), l_{k}, l_{l}\right)$ instruction when there is no object $a_{r}$ is present in the environment, that is, when register $r$ is empty. In this case, similarly to the previous one, we either get the objects $l_{k} l_{k}$ in the cell $C_{1}$, or the computation will not be able to halt.

\begin{tabular}{|c|cccc|ccc|}
\hline & \multicolumn{5}{|c|}{ configuration of $\Pi$} & \multicolumn{3}{c|}{ rules to be applied } \\
& $C_{1}$ & $C_{2}$ & $C_{3}$ & $E n v$ & $P_{1}$ & $P_{2}$ & $P_{3}$ \\
\hline 1. & $l_{j} l_{j}$ & $e e$ & $?$ & $w$ & $j_{1}$ & - & $?$ \\
2. & $l_{j}^{\prime} l_{j}^{\prime}$ & $e e$ & $?$ & $l_{j} w$ & $j_{2}$ & $j_{1}$ & $?$ \\
$3 \cdot$ & $l_{j}^{\prime \prime} l_{j}^{\prime \prime}$ & $l_{j} e$ & $e e$ & $l_{j}^{\prime} w$ & $j_{3}$ & - & $j_{1}$ \\
$4 \cdot$ & $l_{j}^{\prime \prime \prime} l_{j}^{i v}$ & $l_{j} e$ & $l_{j}^{\prime} T_{1}$ & $l_{j}^{\prime \prime} w$ & $j_{4} / j_{5}$ & $j_{3}$ & $j_{2}$ \\
$5 \cdot$ & $\bar{l}_{k} \bar{l}_{k} / \bar{l}_{l} \bar{l}_{l}$ & $l_{j}^{\prime \prime} e$ & $e T_{2}$ & $\left(l_{j}^{\prime \prime \prime} / l_{j}^{i v}\right) w$ & $j_{6} / j_{8}$ & $j_{9} / j_{11}$ & - \\
6. & $\bar{l}_{k} \bar{l}_{k} / \bar{l}_{l} \bar{l}_{l}$ & $l_{j}^{\prime \prime \prime} K / l_{j}^{i v} e$ & $e T_{2}$ & $\left(\bar{l}_{k} / \overline{l_{l}}\right) w$ & $j_{7} / j_{9}$ & $j_{10} / j_{12}$ & $j_{5, k} / j_{5, l}$ \\
\hline $7 \cdot$ & $l_{k} l_{k} / l_{l} l_{l}$ & $e K / e e$ & $\left(\bar{l}_{k} / \bar{l}_{l}\right) T_{4}$ & $\left(\overline{\bar{l}}_{k} / \overline{\bar{l}}_{l}\right) w$ & $k_{1} / l_{1}$ & $j_{8} /-$ & $j_{6, k} / j_{6, l}$ \\
8. & $l_{k}^{\prime} l_{l}^{\prime} / l_{l}^{\prime} l_{l}^{\prime}$ & $e K / e e$ & $\left(\overline{\bar{l}}_{k} / \overline{\bar{l}}_{l}\right) T_{5}$ & $\left(l_{k} / l_{l}\right) w$ & $k_{2} / l_{2}$ & $j_{8} / k_{1}$ & $j_{7, k} / j_{7, l}$ \\
$9 \cdot$ & $l_{k}^{\prime \prime} l_{k}^{\prime \prime \prime} / l_{l}^{\prime \prime} l_{l}^{\prime \prime}$ & $e K /\left(l_{k} / l_{l}\right) e$ & $e e$ & $\left(l_{k}^{\prime} / l_{l}^{\prime}\right) w$ & $k_{3} / l_{3}$ & $j_{8} / k_{2}$ & $j_{1}$ \\
\hline
\end{tabular}

The rules to be applied and the objects contained by the cell $C_{3}$ in row 1 . and row 2 . of the tables above depend on the instruction $l_{i}$ which was simulated before $l_{j}$. If $l_{i}$ is an ADD instruction, then we have $l_{i} T_{1}$ in the first row, and applying the program $i_{2}$ from $P_{3}$ we get $e e$ in the second row, where no program is applied until the next step. Also, $w=w^{\prime}=w^{\prime \prime}$ in this case.

If $l_{i}$ is a SUB instruction, then (as we can also see from row 7. and row 8.) the contents of the cell $C_{3}$ is $\bar{l}_{j} T_{4}$ and $\bar{l}_{j} T_{5}$ in the first two rows where the programs $i_{6, j}$ and $i_{7, j}$ are applied. In this case $w^{\prime \prime}=\overline{\bar{l}}_{j} w$, and $w^{\prime}=w$.

As we have seen above, the $\mathrm{P}$ colony successfully simulates each instruction of $M$ and since there is no program to process $l_{h}$, the label of the halt instruction, it also halts when the computation of $M$ is finished. It is also easy to see that $M$ and $\Pi$ compute the same set of vectors of non-negative integers.

\section{Conclusion}

We have examined extremely simplified variants of P colonies: P colonies of capacity one with no checking rules, and $\mathrm{P}$ colonies with capacity two, but only with senders and consumers. We have shown that even these very simple variants are able to simulate arbitrary register machines, that is, to compute all Turing computable sets of vectors. 


\section{Bibliography}

[1] L. Cienciala, L. Ciencialová, A. Kelemenová. On the number of agents in P colonies. In: Membrane Computing. 8th International Workshop, WMC 2007. Thessaloniki, Greece, June 25-28, 2007. Revised Selected and Invited Papers. Edited by G. Eleftherakis, P. Kefalas, Gh. Păun, G. Rozenberg, A. Salomaa. Volume 4860 of Lecture Notes in Computer Science, Springer-Verlag, Berlin-Heidelberg, 2007, 193-208.

[2] E. Csuhaj-Varjú, J. Dassow, J. Kelemen, Gh. Păun. Grammar Systems - A Grammatical Approach to Distribution and Cooperation. Gordon and Breach, London, 1994.

[3] E. Csuhaj-Varjú, J. Kelemen, A. Kelemenová, Gh. Păun, Gy. Vaszil. Computing with cells in environment: P colonies. Journal of Multi-Valued Logic and Soft Computing 12:201-215, 2006.

[4] J. Kelemen, A. Kelemenová. A grammar-theoretic treatment of multi-agent systems. Cybernetics and Systems 23:621-633, 1992.

[5] J. Kelemen, A. Kelemenová, Gh. Păun. Preview of P colonies: A biochemically inspired computing model. In: Workshop and Tutorial Proceedings. Ninth International Conference on the Simulation and Synthesis of Living Systems (Alife IX). Edited by M. Bedau et al. Boston Mass., 2004, 82-86.

[6] M. Minsky. Computation - Finite and Infinite Machines. Prentice Hall, Englewood Cliffs, NJ, 1967.

[7] Gh. Păun. Membrane Computing - An Introduction. Springer-Verlag, Berlin, 2002.

Lucie Ciencialová has received her $\mathrm{PhD}$. in 2008 and she works as an assistant professor at the Institute of Computer Science, Silesian University in Opava. Her interests include theoretical informatics and natural computing.

Erzsébet Csuhaj-Varjú D.Sc, dr. Habil., is head of the Theoretical Computer Science Research Group at the Computer and Automaton Research Institute of the Hungarian Academy of Sciences. She has also been affiliated with the Department of Algorithms and Applications of the Eötvös Loránd University, Budapest, Hungary, as science advisor. Her main research interests are formal languages, distributed systems, and nature-motivated computing. In these areas she has more than 150 papers, a monograph, and eleven edited volumes published in international publication forums.

Alica Kelemenová is an associated professor at the Institute of Computer Science, Silesian University in Opava, Czech republic and at the Department of Computer Science Catholic University in Ružomberok, Slovakia. Her main research interest is theoretical computer science, especially formal language theory and biologically motivated generative devices like L systems and P systems.

György Vaszil, PhD. is a senior research fellow at the Theoretical Computer Science Research Group of the Computer and Automation Research Institute of the Hungarian Academy of Sciences. His research interests are formal language and automata theory, nature motivated computational models. 